\title{
Beta- and Novel Delta-Coronaviruses Are Identified from Wild Animals in the Qinghai-Tibetan Plateau, China
}

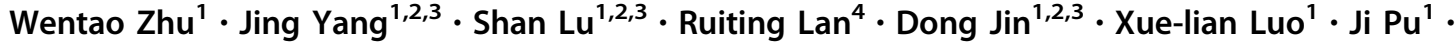 \\ Shusheng $\mathrm{Wu}^{5} \cdot$ Jianguo $\mathrm{Xu}^{1,2,3,6}$
}

Received: 3 August 2020 / Accepted: 27 September 2020 / Published online: 1 December 2020

(c) Wuhan Institute of Virology, CAS 2020

\begin{abstract}
Outbreaks of severe virus infections with the potential to cause global pandemics are increasingly concerning. One type of those commonly emerging and re-emerging pathogens are coronaviruses (SARS-CoV, MERS-CoV and SARS-CoV-2). Wild animals are hosts of different coronaviruses with the potential risk of cross-species transmission. However, little is known about the reservoir and host of coronaviruses in wild animals in Qinghai Province, where has the greatest biodiversity among the world's high-altitude regions. Here, from the next-generation sequencing data, we obtained a known beta-coronavirus (beta-CoV) genome and a novel delta-coronavirus (delta-CoV) genome from faecal samples of 29 marmots, 50 rats and 25 birds in Yushu Tibetan Autonomous Prefecture, Qinghai Province, China in July 2019. According to the phylogenetic analysis, the beta-CoV shared high nucleotide identity with Coronavirus HKU24. Although the novel delta-CoV $(\mathrm{MtCoV})$ was closely related to Sparrow deltacoronavirus ISU42824, the protein spike of the novel delta-CoV showed highest amino acid identity to Sparrow coronavirus HKU17 (73.1\%). Interestingly, our results identified a novel host (Montifringilla taczanowskii) for the novel delta-CoV and the potential cross-species transmission. The most recent common ancestor (tMRCA) of MtCoVs along with other closest members of the species of Coronavirus HKU15 was estimated to be 289 years ago. Thus, this study increases our understanding of the genetic diversity of beta-CoVs and delta$\mathrm{CoVs}$, and also provides a new perspective of the coronavirus hosts.
\end{abstract}

Keywords Coronavirus · Qinghai-Tibetan plateau · Rat · Montifringilla taczanowskii · Marmot

Jianguo Xu

xujianguo@icdc.cn

1 State Key Laboratory of Infectious Disease Prevention and Control, National Institute for Communicable Disease Control and Prevention, Chinese Center for Disease Control and Prevention, Beijing 102206, China

2 Shanghai Public Health Clinical Center, Fudan University, Shanghai 201508, China

3 Research Units of Discovery of Unknown Bacteria and Function, Chinese Academy of Medical Sciences, Beijing 100730, China

4 School of Biotechnology and Biomolecular Sciences, University of New South Wales, Sydney, NSW 2052, Australia

5 Yushu Prefecture Center for Disease Control and Prevention, Yushu 815000, China

6 Department of Laboratorial Science and Technology \& Vaccine Research Center, School of Public Health, Peking University, Beijing 100191, China

\section{Introduction}

Coronaviruses (CoVs) are enveloped, positive-sense and single-stranded RNA viruses that infect humans and animals, which can cause enteric, hepatic and neurological diseases (Wang et al. 2015; Shi et al. 2016; Ge et al. 2017). Several of them are notorious etiological agents and have leaded to epidemic, such as SARS-CoV and MERS-CoV (Drosten et al. 2003; Zaki et al. 2012). In 2020, CoVs have aroused unprecedented public concern due to the ongoing COVID-19 pandemic caused by SARS-CoV-2 (Phan et al. 2020; Wu et al. 2020; Zhou et al. 2020). Due to the unique viral replication process, CoVs have a high mutation rate and recombination frequency (Lai and Cavanagh 1997). These features increase the possibility for CoVs to adapt to novel hosts and environments (Herrewegh et al. 1998; Woo et al. 2006; Lau et al. 2012, 2016; Moreno et al. 2017), 
having a potential of avian-to-mammalian and avian-toavian transmission (Lau et al. 2018).

$\mathrm{CoV}$ s belonging to the family Coronaviridae were divided into four genera: Alpha-CoV, Beta-CoV, Gamma-CoV and Delta-CoV (https://talk.ictvonline.org/taxonomy/). Generally, bats are the reservoirs of Alpha-CoV and Beta$\mathrm{CoV}$, and birds are the reservoirs of Gamma-CoV and DeltaCoV (Woo et al. 2012; Lau et al. 2015; Xu et al. 2016). The genus Delta-CoV contains seven species (https://talk.ictvon line.org/taxonomy/) from both mammals and birds (Woo et al. 2012; Lau et al. 2018). Porcine CoV HKU15, now commonly referred to as porcine deltacoronavirus (PDCoV), has been identified as one of the major enteric pathogen to pigs causing diarrhea accompanied by vomiting, dehydration, loss of weight and death (Wang et al. 2014; Chen et al. 2015; Jung et al. 2015; Ma et al. 2015). Together with PDCoV, sparrow CoV (SpCoV) HKU17 and four SpCoVs (ISU690-4, ISU690-7 ISU42824 and ISU73347) belong to the species of Coronavirus HKU15 in genus Delta-CoV (Chen et al. 2018; Lau et al. 2018).

Marmot (Marmota himalayana) was identified as a major host of Yersinia pestis in Qinghai-Tibet plateau of China (Ge et al. 2015) and has been continuously monitored under China's Plague Surveillance Program. Several of our recent studies have found the possibility of marmots to be the host and reservoir for other pathogens including a new subtype of tick-borne encephalitis virus and novel bisegmented and unsegmented picobirnaviruses (Dai et al. 2018; Luo et al. 2018).

Considering the highly-adaptive and potential pathogenic nature of coronaviruses, we collected samples from various wild animals from Yushu Tibetan Autonomous Prefecture, Qinghai Province, where has the greatest biodiversity among the world's high-altitude regions. According to the next-generation sequencing data and phylogenetic analysis, we identified a novel delta-CoV from wildlife samples and a novel host Montifringilla taczanowskii.

\section{Materials and Methods}

\section{Sample Collection}

In July 2019, 29 marmots and 50 rats were obtained from Yushu Tibetan Autonomous Prefecture in Qinghai Province of China, and corresponding faecal samples were collected. Twenty-five birds near the plateau pika holes from different sites were also obtained while acquiring plateau pikas. Their faecal samples were collected. All the samples were preserved in viral transport medium (Lau et al. 2015) and transferred to our lab in Beijing by cold chain transportation and stored at $-80{ }^{\circ} \mathrm{C}$. Marmots and rats were identified by professionals through morphological observation. Mitochondrial cytochrome b $(C y t b)$ gene was used to identify bird species (Sorenson et al. 1999; Saetre et al. 2001). All sampling work was conducted as part of plague surveillance in animals carried out by Yushu Prefecture Center for Disease Control and Prevention.

\section{RNA Extraction and Next-Generation Sequencing}

The RNA was extracted from each faecal sample using QIAamp viral RNA mini kit (Qiagen, Germany). Total RNA from rats, marmots and birds was grouped into three separate libraries for Illumina HiSeq2000 sequencing. After removing adapters, low-quality reads and host/rRNA sequences, high-quality (clean) data was applied to de novo assembly using Trinity version 2.4.0 (Grabherr et al. 2011) and annotated using BLASTx search in non-redundant protein database. To verify the assembled genomes, reads of clean data were mapped back to the obtained almost complete genomes of coronaviruses using Hisat 2 version 2.1 (Kim et al. 2015), respectively.

\section{Prevalence Screening of Identified Coronavirus in Animal Samples}

To screen the prevalence of coronaviruses in corresponding samples, the $954 \mathrm{bp}$ fragment of delta-CoV obtained in this study (delta-F1: GCTACGGAACGACCTGGGAT; deltaR1: ATTGGTTTGCGTCTGAGGTGA) and 440 bp fragment of beta-CoV (beta-F1: GGTTGGGATTATCCTAA GTGCGA; beta-R1: ACCATCATCACTCAAAATCA TCA) based on RNA-dependent RNA polymerase gene $(R d R p)$ were respectively amplified by PrimeScript ${ }^{\mathrm{TM}}$ One Step RT-PCR Kit Ver.2 (Takara, Japan). PCR products were examined using $1.5 \%$ agarose electrophoresis and Sanger sequenced.

\section{Phylogenetic Analysis}

Sequences were aligned using Mafft version 7 (Katoh and Standley 2013). Based on Akaike information criterion 1 (AIC1), best fit nucleotide substitution models of genome $(\mathrm{GTR}+\mathrm{I}+\mathrm{G})$, OFR1ab $(\mathrm{LG}+\mathrm{G}+\mathrm{F}), \quad \mathrm{S} \quad(\mathrm{WAG}+$ $\mathrm{G}+\mathrm{F}), \mathrm{M}(\mathrm{LG}+\mathrm{G})$ and $\mathrm{N}(\mathrm{LG}+\mathrm{G}+\mathrm{F})$ analyses were conducted using Model Generator version 0.57 (Keane et al. 2006). Phylogenetic trees were reconstructed using PhyML 3.0 (Guindon et al. 2010) and visualized in Tree of Life version 1.0 (Letunic and Bork 2016).

\section{Recombination Analysis}

Genome sequences of thrush coronavirus (ThCoV) HKU12, munia coronavirus (MunCoV) HKU13, SpCoV 
ISU690-4 and Montifringilla taczanowskii CoV HM (obtained in this study, as query sequence) were aligned using Mafft version 7 (Katoh and Standley 2013). Bootscan analysis in SimPlot version 3.5.1 (Lole et al. 1999) was used to detected the possible recombination events. Parameters were set as step $200 \mathrm{bp}$, window size 1,000 bp and model F84.

\section{Divergence Dates Analysis}

The RdRp nucleotide sequences of genus delta-CoV were aligned. Divergence dates were estimated using BEAST version 1.10.4 (Suchard et al. 2018). The most recent common ancestor (tMRCA) was calculated using GTR + $\mathrm{G}+\mathrm{I}$ substitution model and uncorrelated relaxed clock type with log-normal relaxed distribution. The Bayesian Markov chain Monte Carlo was run for $2 \times 10^{8}$ generations with a sampling frequency of every 1,000 steps. The ESS values of parameters should be greater than 200 and visualized using Tracer version 1.7.1. The tMRCA tree was annotated using TreeAnnotator version 1.10.4 in BEAST and visualized in FigTree version 1.4.4 (Suchard et al. 2018).

\section{Nucleotide Sequence Accession Numbers}

The genomes of CoVs obtained in this study were deposited in GenBank with accession numbers: MT215337, MT215336 and MT430884, respectively.

\section{Results}

\section{Identification of Beta-CoV and the Novel Delta- CoV}

One full-length genome $(31,270 \mathrm{bp})$ related to beta-CoV was acquired from the pool of rats. To verify the assembled contig, a total of 64,612 reads $(0.62 \%)$ were mapped to the beta-CoV genome. The identified genome (named as Apodemus peninsulae $\mathrm{CoV}$, accession numbers: MT430884) shared 94.5\%-95.3\% nucleotide (nt) identities with members of the species of Coronavirus HKU24. There was over $90 \%$ amino acid similarity regarding potential structural and non-structural proteins between Apodemus peninsulae $\mathrm{CoV}$ and closely related CoVs. To screen the prevalence of Apodemus peninsulae $\mathrm{CoV}$ in samples, we amplified the $440 \mathrm{bp}$ fragment and the results showed that three out of fifty $(6.0 \%)$ rat samples were positive (Table 1). For positive samples, two of them were Apodemus peninsulae and the other one was Microtus gregalis.

Two mostly identical genomes were identified to be related to delta-CoV. They were from the marmot pool and the bird pool, which were named as Montifringilla taczanowskii CoV (MtCoV) HM (MT215337) and MtCoV N (MT215336), respectively. The numbers of reads mapped to genomes of $\mathrm{MtCoV} \mathrm{HM}$ and $\mathrm{MtCoV} \mathrm{N}$ were 18,676 $(0.01 \%)$ and $10,854(0.01 \%)$, respectively. The prevalence of $\mathrm{MtCoV}$ in marmots and birds were $3.4 \%(1 / 29)$ and

Table 1 Prevalence of beta- and delta-CoV in animal samples collected in Qinghai-Tibetan Plateau in July 2019.

\begin{tabular}{lllll}
\hline Name & Accession number & Sample types & $\begin{array}{l}\text { No. of positive samples/no. of } \\
\text { test samples (\% positive) }\end{array}$ & Detected coronaviruses \\
\hline Apodemus peninsulae CoV & MT430884 & Rats & $3 / 50(6.0)$ & Betacoronavirus \\
MtCoV N & MT215336 & Birds & $4 / 25(16.0)$ & Deltacoronavirus \\
MtCoV HM & MT215337 & Marmots & $1 / 29(3.4)$ & Deltacoronavirus \\
\hline
\end{tabular}

Fig. 1 Comparision of genome organization of $\mathrm{MtCoV} \mathrm{N}$, PorCoV HKU15, and SpCoV HKU17.

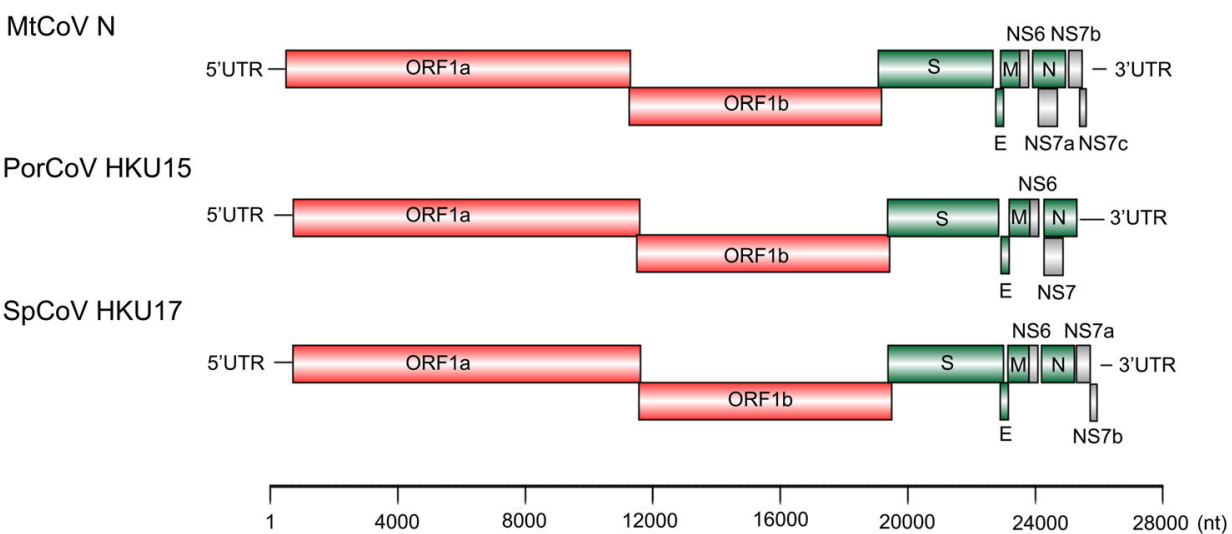


Table 2 Potential codings and predicted transcription regulatory sequences of the genome of MtCoV.

\begin{tabular}{lrrlll}
\hline MtCoV & Location $(\mathrm{nt})$ & Length $(\mathrm{aa})$ & Frame(s) & TRS location & TRS sequence distance bases to AUG \\
\hline ORF1ab & $497-19,227$ & 6,242 & $+2,+1$ & 37 & ACACCA(453)AUG \\
$\mathrm{S}$ & $19,209-22,808$ & 1,199 & +3 & 19,062 & ACACCA(140)AUG \\
$\mathrm{E}$ & $22,802-23,053$ & 83 & +2 & 22,775 & ACCCCA(20)AUG \\
M & $23,046-23,699$ & 217 & +3 & 23,019 & ACACCA(20)AUG \\
NS6 & $23,699-23,980$ & 93 & +2 & 23,646 & ACACCA(46)AUG \\
N & $24,005-25,033$ & 342 & +2 & 23,991 & ACACCA(7)AUG \\
NS7a & $24,099-24,695$ & 198 & +3 & 23,991 & ACACCA(101)AUG \\
NS7b & $25,044-25,472$ & 142 & +3 & 25,033 & ACACCA(4)AUG \\
NS7c & $25,394-25,588$ & 64 & +2 & 25,348 & ACACGA(39)AUG \\
\hline
\end{tabular}

Table 3 Comparison of amino acid identities between MtCoV and closely related CoVs.

\begin{tabular}{|c|c|c|c|c|c|}
\hline & Domain & SpCoV HKU17 & PorCoV HKU15 & SpCoV ISU690-4 & QuaCoV UAE-HKU30 \\
\hline \multicolumn{6}{|c|}{ Amino acid identity (\%) } \\
\hline \multirow[t]{12}{*}{$\mathrm{MtCoV}$} & ADRP & 94.5 & 95.3 & 93.8 & 89.8 \\
\hline & $3 \mathrm{CL}^{\mathrm{pro}}$ & 90.8 & 88.6 & 91.5 & 89.9 \\
\hline & $\operatorname{RdRp}$ & 95.0 & 94.9 & 94.7 & 93.2 \\
\hline & Hel & 97.8 & 97.3 & 97.7 & 97.5 \\
\hline & ExoN & 95.9 & 94.8 & 95.4 & 94.6 \\
\hline & NendoU & 90.5 & 88.4 & 89.6 & 89.9 \\
\hline & O-MT & 91.4 & 90.3 & 92.5 & 92.8 \\
\hline & Concatenated & 94.5 & 93.7 & 94.3 & 93.4 \\
\hline & S & 73.1 & 44.8 & 45.3 & 45.4 \\
\hline & $\mathrm{E}$ & 84.3 & 83.1 & 83.1 & 83.1 \\
\hline & M & 87.1 & 87.1 & 86.6 & 87.1 \\
\hline & $\mathrm{N}$ & 90.4 & 88.9 & 89.8 & 88.6 \\
\hline
\end{tabular}

$16.0 \%$ (4/25), respectively (Table 1). Among positive samples, the four birds were Montifringilla taczanowskii, and the marmot was Marmota himalayana.

\section{Genome Characterization of the Novel Delta-CoV}

The two delta-CoV genomes were of the same length of $25,896 \mathrm{nt}$ with $41.3 \% \mathrm{G}+\mathrm{C}$ content and only had four nucleotide differences between each other at locations 2,254, 5,761, 10,826 and 19,787. The first two base changes $(2,254$ and 5,761) were synonymous changes, the third was in a noncoding region and the last $(19,787)$ was a nonsynonymous change in the protein spike (proline in $\mathrm{MtCoV} \mathrm{HM}$ and serine in $\mathrm{MtCoV} \mathrm{N}$ ). The genome structure of $\mathrm{MtCoV} \mathrm{HM}$ and $\mathrm{MtCoV} \mathrm{N}$ (Fig. 1) shared high similarity with those of PDCoV and SpCoV HKU17 (Woo et al. 2012), including $5^{\prime}$ UTR (untranslated region), replicase ORF1ab, spike (S), envelope (E), membrane (M), nonstructural protein 6 (NS6), nucleocapsid (N), NS7a, NS7b, NS7c, and $3^{\prime}$ UTR (Fig. 1). The putative transcription regulatory sequence (TRS) was identified based on the motif 5'-ACACCA-3' (Table 2). Interestingly, the distance between the TRS and the first base of the initiation codon of ORF NS7a is $101 \mathrm{bp}$, which is the longest compared with those of six members of the genus Delta-CoV that contained a NS7a gene (Woo et al. 2012), which ranged from 4 to $80 \mathrm{bp}$.

MtCoV was identified as a novel member of the species. Pairwise nucleotide sequence alignment of the novel $\mathrm{MtCoV}$ genome showed the highest homologies to $\mathrm{SpCoV}$ ISU690-4 (83.3\%), followed by SpCoV HKU17 (83.0\%), QuaCoV UAE-HKU30 (78.5\%) and PorCoV HKU15 $(82.8 \%)$. The amino acid identities of ADRP, 3CL ${ }^{\text {pro }}$, RdRp, Hel, ExoN, NendoU and O-MT between MtCoV and their closely related strains were summarized in Table 3. Results showed that the concatenated seven 


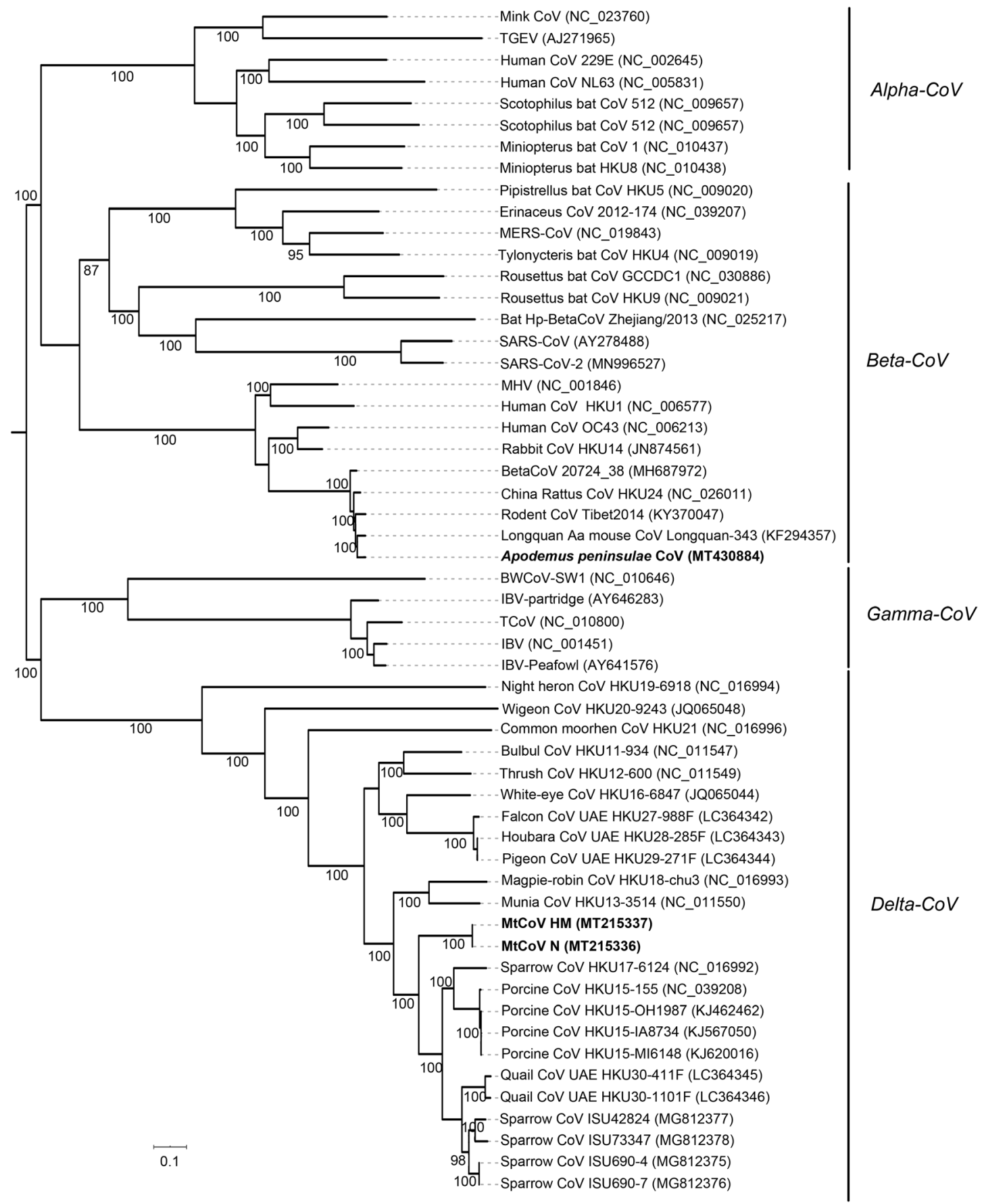

Fig. 2 Phylogenetic analysis of genome sequences of coronaviruses. Bootstrap values $(\geq 70 \%)$ are showed along branches. Scale bar suggests nucleotide substitutions per site.

replicase domains revealed more than $90 \%$ amino acid identity to the members of species of Coronavirus HKU15 (Table 3), which suggested that MtCoV belongs to this species (Lau et al. 2018). However, the structural proteins $\mathrm{E}, \mathrm{M}$ and $\mathrm{N}$ in $\mathrm{MtCoV}$ showed lower identities $(83.1 \%-$ $84.3 \%, 86.6 \%-87.1 \%$ and $88.6 \%-90.4 \%$, respectively) to SpCoV HKU17, SpCoV ISU690-4, QuaCoV UAE-HKU30 and PorCoV HKU15. In particular, protein $\mathrm{S}$ in $\mathrm{MtCoV}$ shared the highest amino acid identities to SpCoV HKU17 (73.1\%), following with Houbara coronavirus (HouCoV) UAE-HKU28 (72.2\%), Pigeon coronavirus (PiCoV) UAEHKU29 (72.1\%) and Falcon coronavirus (FalCoV) UAEHKU27 (72.1\%), but very low identities to other members of the same species $(44.8 \%-45.4 \%)$. Overall, these lower identities of structural proteins between $\mathrm{MtCoV}$ and other 


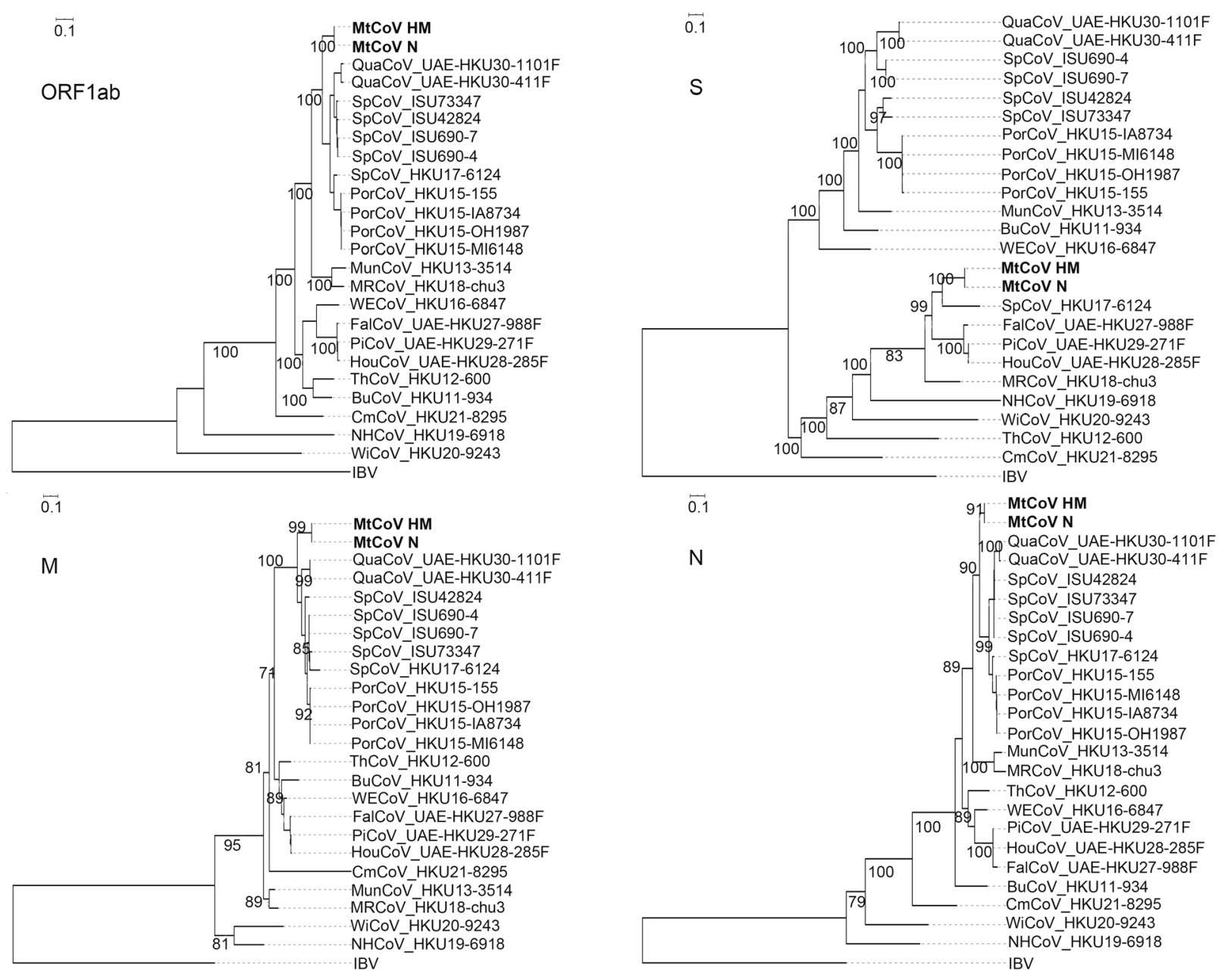

Fig. 3 Phylogenetic tree analyses based on amino acid sequences of ORF1ab, S, M and N of coronaviruses. Bootstrap values ( $\geq 70 \%$ ) are showed along branches. Scale bar suggests nucleotide substitutions per site. Bold strains are the novel ones isolated in this study.

members of the species of Coronavirus HKU15 indicated that $\mathrm{MtCoV}$ represents a novel member in that species.

\section{Phylogenetic Analyses of the Novel Delta-CoV}

Phylogenetic analysis of whole-genome nucleotide sequences showed Apodemus peninsulae CoV was closely related to the cluster including members of the species of Coronavirus HKU24 (Fig. 2). Also, the result further confirmed that MtCoV belongs to the genus Delta-CoV and forms an independent lineage. It was closely related to PorCoV HKU15 and SpCoV HKU17 (Fig. 2). Further, phylogenetic analyses based on amino acid sequences of proteins ORF1ab, $\mathrm{M}$ and $\mathrm{N}$ were identical to trees based on nucleotide sequences, and both revealed that $\mathrm{MtCoV} \mathrm{HM}$ and $\mathrm{MtCoV} \mathrm{N}$ were clustered with members of the species of Coronavirus HKU15 but in the meantime different from them (Fig. 3). The protein $\mathrm{S}$ based phylogenetic tree showed that MtCoVs were grouped with SpCoV HKU17, and were more closely related to FalCoV UAE-HKU27,
PiCoV UAE-HKU29, HouCoV UAE-HKU28 and magpie robin coronavirus (MRCoV) HKU18 (Fig. 3). It was due to the high identities of protein $\mathrm{S}$ and was consistent with previous reports (Woo et al. 2012; Chen et al. 2018).

\section{Recombination Analysis of Delta-CoV}

The genomes of ThCoV HKU12, MunCoV HKU13, SpCoV ISU690-4 and MtCoV HM (as query sequence) were aligned for recombination analysis using Bootscan. The result indicated the potential long recombination segment from aligned positions 19,500 to 23,300 , which were mainly located in the $S$ gene of $\mathrm{MtCoV}$ (Fig. 4A). The recombinant segment was likely to be derived from $\mathrm{ThCoV}$ HKU12. Since the receptor-binding domain (RBD) locates in the $\mathrm{S}$ protein, the recombined sequence might lead to biological changes of receptor binding and thus initiate cross-species transmission. Two short potential recombination segments were also found in aligned positions 11,300 to 11,700 and 15,500 to 16,100 of $\mathrm{MtCoV}$ 
A

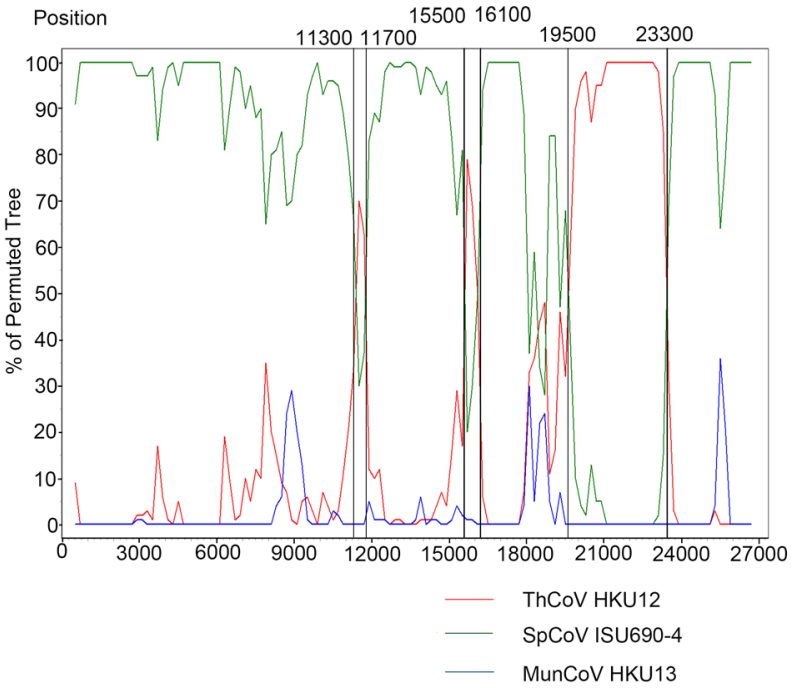

Fig. 4 Potential recombination event detected using bootscan analysis. A Genome of MtCoV HM was used as the query sequence and compared with ThCoV HKU12, MunCoV HKU13 and SpCoV
B

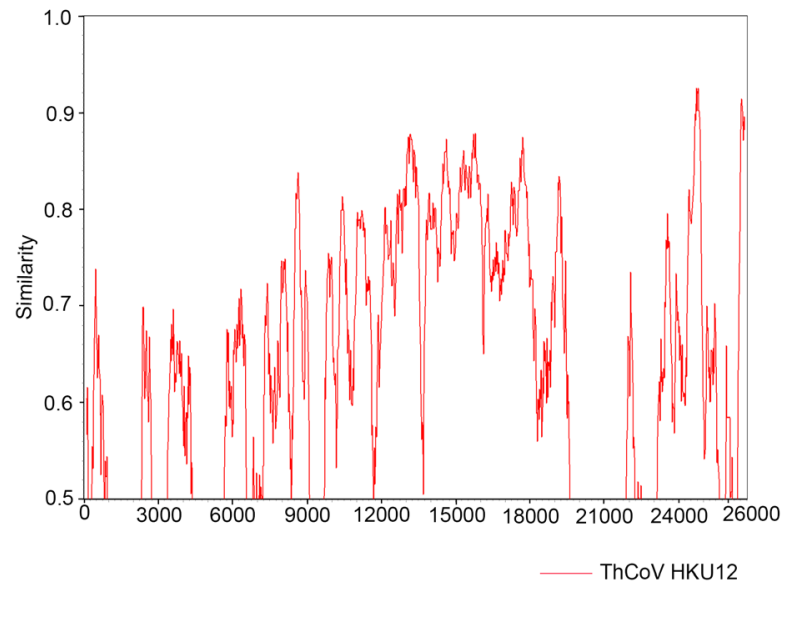

ISU690-4. Red lines indicated the recombination sites. B MtCoV HM was used as the query sequence and compared with the genome of ThCoV-HKU12.

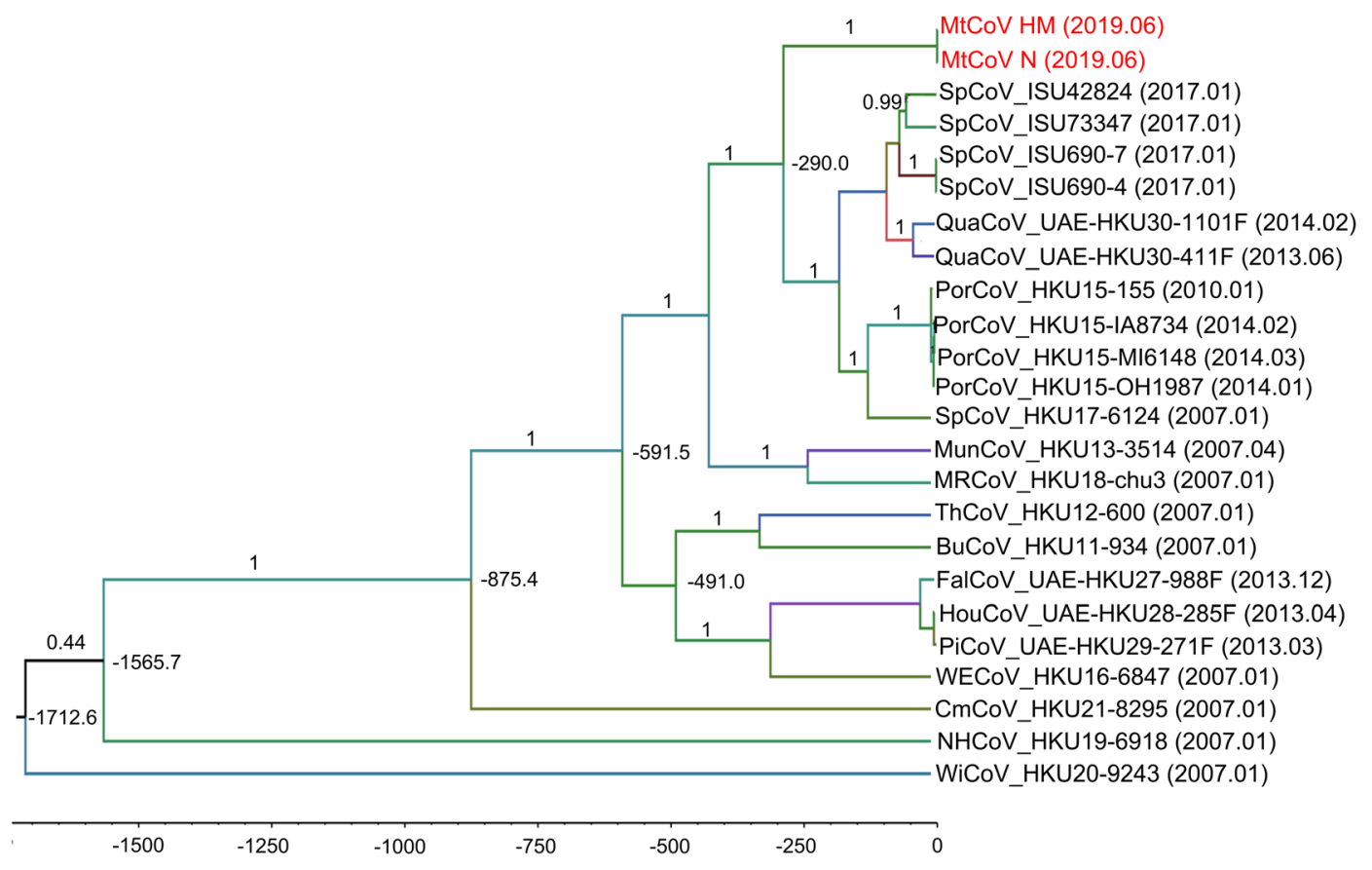

Fig. 5 Bayesian Markov chain Monte Carlo (MCMC) tree analysis of the novel delta-CoV based on RdRp nucleotide sequences. The posterior probabilities and tMRCA are showed on branch labels and node labels. Sampling date is marked on the end of tip labels.

(Fig. 4A). Genome sequence of MtCoV HM (as the query sequence) was compared with that of ThCoV-HKU12 using Simplot analysis (Fig. 4B). Result from global align in BLAST indicated that nucleotide identity values of $S$ gene between MtCoV HM (19,209 to 22,808 nt) and ThCoV-HKU12 (19,433 to 23,011 nt) was $58.5 \%$, with part of $S$ gene of MtCoVs showed above $70 \%$ nucleotide identity to ThCoV-HKU12 (Fig. 4B). And, $S$ gene of MtCoVs shared higher nucleotide identity with $\mathrm{PiCoV}$ UAE-HKU29 (73.3\%), HouCoV UAE-HKU28 (73.3\%), FalCoV UAEHKU27 (73.2\%), SpCoV HKU17 (71.0\%), Magpie robin coronavirus HKU18 (67.4\%), Night heron coronavirus HKU19 (58.7\%) and Wigeon coronavirus HKU20 (58.6\%). 


\section{Estimation of Divergence Dates of Delta-CoV}

To estimate the divergence time of $\mathrm{MtCoV}$, the $R d R p$ nucleotide sequences of genus delta-CoV were selected (Lau et al. 2018) and aligned to calculate tMRCA using GTR + G + I substitution model and uncorrelated relaxed clock type with the log-normal relaxed distribution. The result of molecular clock analysis indicated that the most recent common ancestor of $\mathrm{MtCoVs}$ along with other closest members of the species in Coronavirus HKU15 was estimated to be $289(95 \%$ HPD $=60-1140)$ years ago (Fig. 5).

\section{Discussion}

Based on the genome data, we report the characterization of two coronaviruses identified from the wild animal samples in Yushu Tibetan Autonomous Prefecture, Qinghai Province, China.

The concatenated seven replicase domains of Apodemus peninsulae $\mathrm{CoV}$ shared $98.9 \%-99.1 \%$ amino acid identities to the members of species in Coronavirus HKU24. Among them, the beta-CoV was detected from both Apodemus peninsulae and Microtus gregalis in Qinghai, China. The other members of the same species were previously detected from Rattus norvegicus in Guangdong (Lau et al. 2015), Apodemus agrarius in Zhejiang (Wang et al. 2015), Apodemus peninsulae in Tibet (Wu et al. 2018), Apodemus chevrieri in Yunnan (Ge et al. 2017), China and Rattus argentiventer in Vietnam (Phan et al. 2018). Those results indicate the members in Coronavirus HKU24 shared high nucleotide identity and may be commonly present in rats. However, the pathogenic potential remains unknown.

As a member of delta-CoV, PorCoV HKU15 was firstly reported in pigs without pathogenic evidence in Hong Kong of China in 2012, later detected in pig farms causing watery and acute diarrhea in U.S. in 2014, and then in Thailand, the Mainland of China, Korea and Lao PDR with 30\%-40\% death rate (Wang et al. 2014; Chen et al. 2015; Jung et al. 2015; Ma et al. 2015). PorCoV HKU15 shares the most recent common ancestor with $\mathrm{SpCoV}$ strains (HKU17, ISU42824, ISU690-4, ISU690-7 and ISU73347) suggesting the potential of cross-species transmission and/ or shared host reservoir between pigs and sparrows. $\mathrm{MtCoVs}$ belong to the same $\mathrm{CoV}$ species with QuaCoV UAE-HKU30, PorCoV HKU15 and SpCoV HKU17, and all revealed phylogenetic positions shifting in phylogenetic trees based on different genes (Lau et al. 2018). And, species of hosts of Coronavirus HKU15 were diverse, such as quail (order Galliformes, family Phasianidae), sparrows (order Passeriformes, family Ploceidae) and Montifringilla taczanowskii (order Passeriformes, family Passeridae). For $\mathrm{MtCoV}$, almost the entire $S$ gene may be a recombinant from another more distant related delta-CoV. A part of $S$ gene of MtCoVs shared above $70 \%$ nucleotide identity to ThCoV-HKU12 (Fig. 4B). The estimated tMRCA of MtCoV HM and ThCoV-HKU12 was around 600 years ago. So, the low nucleotide identity values of $\mathrm{MtCoV} \mathrm{HM}$ and ThCoV-HKU12 in $S$ gene (full length) indicated that the virus may has evolved to accommodate the new host after recombination (Lau et al. 2018). However, it is worth further investigating their cross-species transmission potential and mechanism.

Interestingly, we found two mostly identical sequences between MtCoV HM and MtCoV N. Firstly, the faecal samples of Montifringilla taczanowskii and Marmota himalayana were collected by Yushu Prefecture Center for Disease Control and Prevention at different times and different sites. Therefore, the cross-contamination of species was ruled out. Secondly, viral RNAs of faecal samples of Montifringilla taczanowskii and Marmota himalayana were extracted on different dates and sequenced as different pools. Thus, cross-contamination of the samples was also ruled out. Montifringilla taczanowskii and Marmota himalayana are common wild animals in Qinghai-Tibet plateau, and Montifringilla taczanowskii can enter marmot and pika caves (Ge et al. 2020). Because only one positive stool samples of Marmota himalayana was detected, the delta-CoV may be present accidentally in intestinal tract of the Marmota himalayana. The mechanism how Marmota himalayana got the delta-CoV still needs further study.

Overall, our study identified a novel delta-CoV and for the first time, found that Montifringilla taczanowskii may be a novel host of delta-CoV. This study facilitates a better understanding of the genetic diversity of delta-CoVs. Because the isolation was unsuccessful, the pathogenic potential of $\mathrm{MtCoV}$ is still unknown and requires further studies.

Acknowledgements This work was supported by grants from National Science and Technology Major Project of China (2018ZX10712001-018, 2017ZX10303405-002, 2017ZX10303405005-002), National Science and Technology of China (2017FY101202), National Key R\&D Program of China (2019YFC1200500 and 2019YFC1200505), Sanming Project of Medicine in Shenzhen (SZSM201811071) and Research Units of Discovery of Unknown Bacteria and Function (2018RU010). The authors thank professor Changyun Ye for sharing the samples of rats.

Author Contributions JX and WZ conceived this study. WZ, JY, SL, XL, and SW collected the samples. WZ and DJ conducted experiments. WZ, JP and JY performed sequencing and analyzed the data. WZ, JY, and RL drafted the manuscript. JX finalized and supervised the study. All authors read and approved the final version of the manuscript. 


\section{Compliance with Ethical Standards}

Conflict of interest The authors declare that they have no conflict of interest.

Animal and Human Rights Statement The study practices were approved by Ethical Committee of the National Institute for Communicable Disease Control and Prevention, Chinese Center for Disease Control and Prevention (NO: ICDC-2016004).

\section{References}

Chen Q, Gauger P, Stafne M, Thomas J, Arruda P, Burrough E, Madson D, Brodie J, Magstadt D, Derscheid R, Welch M, Zhang J (2015) Pathogenicity and pathogenesis of a United States porcine deltacoronavirus cell culture isolate in 5-day-old neonatal piglets. Virology 482:51-59

Chen Q, Wang L, Yang C, Zheng Y, Gauger PC, Anderson T, Harmon KM, Zhang J, Yoon KJ, Main RG, Li G (2018) The emergence of novel sparrow deltacoronaviruses in the United States more closely related to porcine deltacoronaviruses than sparrow deltacoronavirus HKU17. Emerg Microbes Infect 7:105

Dai X, Shang G, Lu S, Yang J, Xu J (2018) A new subtype of eastern tick-borne encephalitis virus discovered in Qinghai-Tibet Plateau. China Emerg Microbes Infect 7:74

Drosten C, Günther S, Preiser W, van der Werf S, Brodt HR, Becker S, Rabenau H, Panning M, Kolesnikova L, Fouchier RA, Berger A, Burguière AM, Cinatl J, Eickmann M, Escriou N, Grywna K, Kramme S, Manuguerra JC, Müller S, Rickerts V, Stürmer M, Vieth S, Klenk HD, Osterhaus AD, Schmitz H, Doerr HW (2003) Identification of a novel coronavirus in patients with severe acute respiratory syndrome. $\mathrm{N}$ Engl $\mathrm{J}$ Med 348:1967-1976

Ge P, Xi J, Ding J, Jin F, Zhang H, Guo L, Zhang J, Li J, Gan Z, Wu B, Liang J, Wang X, Wang X (2015) Primary case of human pneumonic plague occurring in a Himalayan marmot natural focus area Gansu Province, China. Int J Infect Dis 33:67-70

Ge XY, Yang WH, Zhou JH, Li B, Zhang W, Shi ZL, Zhang YZ (2017) Detection of alpha- and betacoronaviruses in rodents from Yunnan. China Virol J 14:98

Ge Y, Yang J, Lai XH, Zhang G, Jin D, Lu S, Wang B, Huang Y, Huang Y, Ren Z, Zhang X, Xu J (2020) Vagococcus xieshaowenii sp. nov., isolated from snow finch (Montifringilla taczanowskii) cloacal content. Int $\mathrm{J}$ Syst Evol Microbiol 70:2493-2498

Grabherr MG, Haas BJ, Yassour M, Levin JZ, Thompson DA, Amit I, Adiconis X, Fan L, Raychowdhury R, Zeng Q, Chen Z, Mauceli E, Hacohen N, Gnirke A, Rhind N, di Palma F, Birren BW, Nusbaum C, Lindblad-Toh K, Friedman N, Regev A (2011) Fulllength transcriptome assembly from RNA-Seq data without a reference genome. Nat Biotechnol 29:644-652

Guindon S, Dufayard JF, Lefort V, Anisimova M, Hordijk W, Gascuel O (2010) New algorithms and methods to estimate maximum-likelihood phylogenies: assessing the performance of PhyML 3.0. Syst Biol 59:307-321

Herrewegh AA, Smeenk I, Horzinek MC, Rottier PJ, de Groot RJ (1998) Feline coronavirus type II strains 79-1683 and 79-1146 originate from a double recombination between feline coronavirus type I and canine coronavirus. J Virol 72:4508-4514

Jung K, Hu H, Eyerly B, Lu Z, Chepngeno J, Saif LJ (2015) Pathogenicity of 2 porcine deltacoronavirus strains in gnotobiotic pigs. Emerg Infect Dis 21:650-654
Katoh K, Standley DM (2013) MAFFT multiple sequence alignment software version 7: improvements in performance and usability. Mol Biol Evol 30:772-780

Keane TM, Creevey CJ, Pentony MM, Naughton TJ, McLnerney JO (2006) Assessment of methods for amino acid matrix selection and their use on empirical data shows that ad hoc assumptions for choice of matrix are not justified. BMC Evol Biol 6:29

Kim D, Langmead B, Salzberg SL (2015) HISAT: a fast spliced aligner with low memory requirements. Nat Methods $12: 357-360$

Lai MM, Cavanagh D (1997) The molecular biology of coronaviruses. Adv Virus Res 48:1-100

Lau SK, Wernery R, Wong EY, Joseph S, Tsang AK, Patteril NA, Elizabeth SK, Chan KH, Muhammed R, Kinne J, Yuen KY, Wernery U, Woo PC (2016) Polyphyletic origin of MERS coronaviruses and isolation of a novel clade A strain from dromedary camels in the United Arab Emirates. Emerg Microbes Infect 5:e128

Lau SK, Woo PC, Li KS, Tsang AK, Fan RY, Luk HK, Cai JP, Chan KH, Zheng BJ, Wang M, Yuen KY (2015) Discovery of a novel coronavirus, China Rattus coronavirus HKU24, from Norway rats supports the murine origin of Betacoronavirus 1 and has implications for the ancestor of Betacoronavirus lineage A. J Virol 89:3076-3092

Lau SK, Woo PC, Yip CC, Fan RY, Huang Y, Wang M, Guo R, Lam CS, Tsang AK, Lai KK, Chan KH, Che XY, Zheng BJ, Yuen KY (2012) Isolation and characterization of a novel Betacoronavirus subgroup A coronavirus, rabbit coronavirus HKU14, from domestic rabbits. J Virol 86:5481-5496

Lau SKP, Wong EYM, Tsang CC, Ahmed SS, Au-Yeung RKH, Yuen KY, Wernery U, Woo PCY (2018) Discovery and sequence analysis of four deltacoronaviruses from birds in the middle east reveal interspecies jumping with recombination as a potential mechanism for avian-to-avian and avian-to-mammalian transmission. J Virol 92:e00265-18

Letunic I, Bork P (2016) Interactive tree of life (iTOL) v3: an online tool for the display and annotation of phylogenetic and other trees. Nucleic Acids Res 44:W242-245

Luo XL, Lu S, Jin D, Yang J, Wu SS, Xu J (2018) Marmota himalayana in the Qinghai-Tibetan plateau as a special host for bi-segmented and unsegmented picobirnaviruses. Emerg Microbes Infect 7:20

Ma Y, Zhang Y, Liang X, Lou F, Oglesbee M, Krakowka S, Li J (2015) Origin, evolution, and virulence of porcine deltacoronaviruses in the United States. mBio 6:e0064

Moreno A, Franzo G, Massi P, Tosi G, Blanco A, Antilles N, Biarnes M, Majó N, Nofrarías M, Dolz R, Lelli D, Sozzi E, Lavazza A, Cecchinato M (2017) A novel variant of the infectious bronchitis virus resulting from recombination events in Italy and Spain. Avian Pathol 46:28-35

Phan LT, Nguyen TV, Luong QC, Nguyen TV, Nguyen HT, Le HQ, Nguyen TT, Cao TM, Pham QD (2020) Importation and humanto-human transmission of a novel coronavirus in Vietnam. N Engl J Med 382:872-874

Phan MVT, Ngo Tri T, Hong Anh P, Baker S, Kellam P, Cotten M (2018) Identification and characterization of Coronaviridae genomes from Vietnamese bats and rats based on conserved protein domains. Virus Evol 4:vey035

Saetre GP, Borge T, Lindell J, Moum T, Primmer CR, Sheldon BC, Haavie J, Johnsen A, Ellegren H (2001) Speciation, introgressive hybridization and nonlinear rate of molecular evolution in flycatchers. Mol Ecol 10:737-749

Shi ZL, Guo D, Rottier PJ (2016) Coronavirus: epidemiology, genome replication and the interactions with their hosts. Virol Sin $31: 1-2$ 
Sorenson MD, Ast JC, Dimcheff DE, Yuri T, Mindell DP (1999) Primers for a PCR-based approach to mitochondrial genome sequencing in birds and other vertebrates. Mol Phylogenet Evol 12:105-114

Suchard MA, Lemey P, Baele G, Ayres DL, Drummond AJ, Rambaut A (2018) Bayesian phylogenetic and phylodynamic data integration using BEAST 110. Virus Evol 4:vey016

Wang L, Byrum B, Zhang Y (2014) Detection and genetic characterization of deltacoronavirus in pigs, Ohio, USA, 2014. Emerg Infect Dis 20:1227-1230

Wang W, Lin XD, Guo WP, Zhou RH, Wang MR, Wang CQ, Ge S, Mei SH, Li MH, Shi M, Holmes EC, Zhang YZ (2015) Discovery, diversity and evolution of novel coronaviruses sampled from rodents in China. Virology 474:19-27

Woo PC, Lau SK, Lam CS, Lau CC, Tsang AK, Lau JH, Bai R, Teng JL, Tsang CC, Wang M, Zheng BJ, Chan KH, Yuen KY (2012) Discovery of seven novel Mammalian and avian coronaviruses in the genus deltacoronavirus supports bat coronaviruses as the gene source of alphacoronavirus and betacoronavirus and avian coronaviruses as the gene source of gammacoronavirus and deltacoronavirus. J Virol 86:3995-4008

Woo PC, Lau SK, Yip CC, Huang Y, Tsoi HW, Chan KH, Yuen KY (2006) Comparative analysis of 22 coronavirus HKU1 genomes reveals a novel genotype and evidence of natural recombination in coronavirus HKU1. J Virol 80:7136-7145
Wu F, Zhao S, Yu B, Chen YM, Wang W, Song ZG, Hu Y, Tao ZW, Tian JH, Pei YY, Yuan ML, Zhang YL, Dai FH, Liu Y, Wang QM, Zheng JJ, Xu L, Holmes EC, Zhang YZ (2020) A new coronavirus associated with human respiratory disease in China. Nature 579:265-269

Wu Z, Lu L, Du J, Yang L, Ren X, Liu B, Jiang J, Yang J, Dong J, Sun L, Zhu Y, Li Y, Zheng D, Zhang C, Su H, Zheng Y, Zhou H, Zhu G, Li H, Chmura A, Yang F, Daszak P, Wang J, Liu Q, Jin Q (2018) Comparative analysis of rodent and small mammal viromes to better understand the wildlife origin of emerging infectious diseases. Microbiome 6:178

Xu L, Zhang F, Yang W, Jiang T, Lu G, He B, Li X, Hu T, Chen G, Feng Y, Zhang Y, Fan Q, Feng J, Zhang H, Tu C (2016) Detection and characterization of diverse alpha- and betacoronaviruses from bats in China. Virol Sin 31:69-77

Zaki AM, van Boheemen S, Bestebroer TM, Osterhaus AD, Fouchier RA (2012) Isolation of a novel coronavirus from a man with pneumonia in Saudi Arabia. N Engl J Med 367:1814-1820

Zhou P, Yang XL, Wang XG, Hu B, Zhang L, Zhang W, Si HR, Zhu Y, Li B, Huang CL, Chen HD, Chen J, Luo Y, Guo H, Jiang RD, Liu MQ, Chen Y, Shen XR, Wang X, Zheng XS, Zhao K, Chen QJ, Deng F, Liu LL, Yan B, Zhan FX, Wang YY, Xiao GF, Shi ZL (2020) A pneumonia outbreak associated with a new coronavirus of probable bat origin. Nature 579:270-273 